\title{
Identification of GSK-3 as a potential therapeutic entry point for epilepsy
}

Najat Aourz $^{1 *}$, Ann-Sophie K. Serruys ${ }^{2 *}$, Joëlle N. Chabwine ${ }^{3}$, Pascal Byenda Balegamire ${ }^{3}$, Tatiana Afrikanova ${ }^{2}$, RuAngelie Edrada-Ebel ${ }^{4}$, Alexander I. Grey ${ }^{4}$, Appolinary R. Kamuhabwa ${ }^{5}$, Laura Walrave ${ }^{1}$, Camila V. Esguerra ${ }^{2}$, Fred van Leuven ${ }^{6}$, Peter A. M. de Witte ${ }^{2}$, Ilse Smolders ${ }^{1 \$}$, Alexander D. Crawford ${ }^{2 \$}$

${ }^{1}$ Center for Neurosciences (C4N), Research Group Experimental Pharmacology (EFAR/FASC), Vrije Universiteit Brussel (VUB), Laarbeeklaan 103, 1090 Brussels, Belgium

${ }^{2}$ Laboratory for Molecular Biodiscovery, Department of Pharmaceutical and Pharmacological Sciences, University of Leuven, Leuven, Belgium

${ }^{3}$ Salama Neuroscience Center, Bukavu, South Kivu, Democratic Republic of the Congo

${ }^{4}$ Strathclyde Institute of Pharmacy \& Biomedical Sciences, University of Strathclyde, Glasgow, Scotland, UK

${ }^{5}$ Department of Pharmacognosy, Muhimbili University of Health \& Allied Sciences, Dar es Salaam, Tanzania

${ }^{6}$ Experimental Genetics Group (LEGTEGG), Department of Human Genetics, University of Leuven, Leuven, Belgium

*equally contributing first authors

\$equally contributing senior authors

This is a peer-reviewed, accepted author manuscript of the following article: Aourz, N., Serruys, A-S. K., Chabwine, J. N., Balegamire, P. B., Afrikanova, T., Edrada-Ebel, R., Grey, A. I., Kamuhabwa, A. R., Walrave, L., Esguerra, C. V., van Leuven, F., de Witte, P. A. M., Smolders, I., \& Crawford, A. D. (2019). Identification of GSK-3 as a potential therapeutic entry point for epilepsy. ACS Chemical Neuroscience, 10(4), 1992-2003. https://doi.org/10.1021/acschemneuro.8b00281 


\section{Corresponding author:}

Najat Aourz

Najat.Aourz@vub.be

$+32.2 .4774429$ 


\begin{abstract}
In view of the clinical need for new antiseizure drugs (ASDs) with novel modes of action, we used a zebrafish seizure model to screen the anticonvulsant activity of medicinal plants used by traditional healers in the Congo for the treatment of epilepsy, and identified a crude plant extract that inhibited pentylenetetrazol (PTZ)-induced seizures in zebrafish larvae. Zebrafish bioassayguided fractionation of this anticonvulsant Fabaceae species, Indigofera arrecta, identified indirubin, a compound with known inhibitory activity of glycogen synthase kinase (GSK)-3, as the bioactive component. Indirubin, as well as the more potent and selective GSK-3 inhibitor 6bromoindirubin-3'-oxime (BIO-acetoxime) were tested in zebrafish and rodent seizure assays. Both compounds revealed anticonvulsant activity in PTZ-treated zebrafish larvae, with electroencephalographic recordings revealing reduction of epileptiform discharges. Both indirubin and BIO-acetoxime also showed anticonvulsant activity in the pilocarpine rat model for limbic seizures and in the 6-Hz refractory seizure mouse model. Most interestingly, BIO-acetoxime also exhibited anticonvulsant actions in 6-Hz fully kindled mice. Our findings thus provide the first evidence for anticonvulsant activity of GSK-3 inhibition, thereby implicating GSK-3 as a potential therapeutic entry point for epilepsy. Our results also support the use of zebrafish bioassay-guided fractionation of anti-epileptic medicinal plant extracts as an effective strategy for the discovery of new ASDs with novel mechanisms of action.
\end{abstract}

Keywords: Epilepsy, 6Hz, indirubin, GSK-3, seizure models, zebrafish 


\section{Introduction}

Most antiseizure drugs (ASDs) developed in the past two decades offer improved therapeutic options for a wide range of epileptic seizures, yet they do not address the problem of pharmacoresistant epilepsy, nor the ability to prevent or cure epilepsy. One third of all epilepsy patients do therefore not adequately respond to any of the existing ASDs.

Over the past decade, the zebrafish has been established as a valuable tool to understand mechanisms involved in the development of seizures and to screen for compounds with anticonvulsant activity in a medium- to high-throughput manner ${ }^{1-3}$. Using both genetic and pharmacological models, zebrafish models of seizures have been shown to share many similarities with their corresponding rodent models, including seizure-like behavior, epileptic discharges, increased $c$-fos expression in the brain and similar responses to AEDs in terms of anticonvulsant $\operatorname{activity}^{4-8}$.

Natural products and their analogues have historically been of considerable value for the discovery and development of pharmaceuticals in numerous indications, including epilepsy ${ }^{9}$. In most developing countries, medicinal plants are still routinely used to treat epileptic seizures ${ }^{10}$, and many of these plant extracts have shown activity in animal models of epilepsy ${ }^{11}$. To explore the potential of medicinal plants for the discovery of novel ASDs, we initiated an effort to identify, using zebrafish seizure models as in vivo bioassays, the anticonvulsant constituents of medicinal plants used for the treatment of epileptic seizures. By using these same zebrafish seizure models for in vivo bioassay-guided fractionation, we have previously reported the isolation of several anticonvulsant secondary metabolites from medicinal plants used in India, China, and the Philippines $^{12-14}$. 
In the current study, we report the identification of indirubin, a glycogen synthase kinase-3 (GSK3) inhibitor, as a novel anticonvulsant compound from the Congolese medicinal plant Indigofera $\operatorname{arrecta}($ I. arrecta).. To explore this potential of medicinal plants for the discovery of novel ASDs, we initiated an effort to identify, using zebrafish seizure models as in vivo bioassays, the antiepileptic constituents of medicinal plants used in Congo for the treatment of epileptic seizures. Furthermore, by using these same zebrafish seizure models for in vivo bioassay-guided fractionation, we have been able to isolate several anticonvulsant secondary metabolites from these extracts $^{12-14}$.

In the current study, we report the identification of indirubin, a Glycogen Synthase Kinase-3 (GSK-3) inhibitor as a novel anticonvulsant compound from the Congolese medicinal plant Indigofera arrecta (I. arrecta). As GSK-3 has not yet been reported as a drug target for epilepsy, we further investigated the antiseizure activity of BIO-acetoxime, an additional GSK-3 inhibitor with higher potency and higher selectivity for GSK-3. Antiseizure activity of both compounds was firstly examined in the pentylenetetrazole (PTZ) zebrafish seizure assay ${ }^{6}$. Moreover, several chemically and electrically induced rodent seizure models were used to further investigate the antiseizure properties of indirubine and BIO-acetoxime. These seizure models include the acute 6Hz corneal mouse model and the pilocarpine rat model. Finally, BIO-acetoxime was also tested in fully 6-Hz corneally kindled mice, an established model of chronic epilepsy ${ }^{15}$. 


\section{Results}

Zebrafish bioassay-guided identification of indirubin as the bioactive component of the medicinal plant I. arrecta

We sought to identify potentially novel anticonvulsant compounds from medicinal plants used by traditional healers in the Congo to treat seizures. For this purpose, we screened methanolic extracts of individual medicinal plants using a larval zebrafish PTZ seizure assay ${ }^{6}$. An extract of the leaves of $I$. arrecta exhibited potent anticonvulsant activity (Figure 1A, extract 3). To isolate and identify the secondary bioactive metabolites of this plant extract (Figure 1B), bioassay-guided fractionation and structural elucidation was performed using high-resolution mass spectrometry and nuclear magnetic resonance, resulting in the identification of indirubin. The spectral data were in agreement with those previously reported for indirubin (data not shown) ${ }^{16-18}$.

\section{Indirubin exerts anticonvulsant effects in three different species in the absence of gross locomotor disturbances or sedation}

Our results showed that $300 \mu \mathrm{M}$ indirubin (Figure 1C) significantly decreased larval locomotor activity induced by PTZ in the behavioral zebrafish assay (Figure 2A), while treatment with $30 \mu \mathrm{M}$ and $100 \mu \mathrm{M}$ alone did not affect larval movement (Figure $2 \mathrm{~B}$ ).

To confirm the seizure-suppressing activity of indirubin, we performed tectal field recordings on zebrafish larvae as previously described ${ }^{1,6}$. EEG recordings showed that $300 \mu \mathrm{M}$ indirubin decreased the number of interictal and ictal-like spikes as well as the total cumulative duration of epileptiform activity (Figure 2C). 
Several rodent seizure models were used to further confirm the anticonvulsant activity of indirubin. Although $2 \mathrm{mg} / \mathrm{kg}$ and $10 \mathrm{mg} / \mathrm{kg}$ indirubin was inactive in the PTZ i.v. tail infusion mouse model (data not shown), these doses did clearly reduce the seizure severity in the focal pilocarpine rat model in a dose-dependent manner (Figure 2D(b)). In addition, dose-dependent anticonvulsant actions of indirubin $(10 \mathrm{mg} / \mathrm{kg}$ and $30 \mathrm{mg} / \mathrm{kg})$ were observed in the acute 6-Hz model for refractory seizures in mice (53 mA stimulation current) (Figure 2E).

To discover possible adverse effects such as motor coordination problems or sedation, the beamwalking assay was performed in mice. Administration of indirubin $(10 \mathrm{mg} / \mathrm{kg})$ did not show any adverse effects. Total time on the beam, the number of foot slips and the number of falls was not different between control animals and indirubin-treated mice (Figure 3A). Diazepam (1 mg/kg, i.p.) was used as a positive control and clearly increased the total time on the beam and the number of falls (Figure 3B).

\section{Roscovitine does not attenuate seizure severity in acute rodent models}

In order to determine the mechanism by which indirubin exerts its anticonvulsant activity in the zebrafish PTZ seizure model, we tested a number of compounds having different affinities for the primary targets of indirubin, i.e. GSK-3, several cyclin dependent kinases (CDKs) and the aryl hydrocarbon receptor (AhR) (Table 1). In general, we can conclude that compounds with greater selectivity towards GSK-3 exhibited clear anticonvulsant activity, while those more selective towards CDK or AhR were inactive. One exception however was roscovitine, a compound that inhibits CDK-5 (and to a lesser extent CDK-2), which was modestly active in the zebrafish PTZ assay. The fact that 1-Azakenpaullone and Aloisine exert a similar protective effect in the zebrafish 
PTZ assay, despite their 50-fold difference in potency, could potentially be explained by differences in bioavailability between the two compounds.

All together, these findings strongly point towards GSK-3 inhibition as the mechanism of action responsible for the anticonvulsant effects of indirubin. However, to rule out the possibility that CDK-5 might be involved in its anticonvulsant actions, we next investigated whether roscovitine (Figure 4A) is able to exert anticonvulsant effects in the focal pilocarpine rat model and the acute 6-Hz mouse model, two rodent models in which indirubin exerted potent anticonvulsant effects. In this regard, we showed that pilocarpine-induced seizures are not attenuated by intrahippocampal perfusion of $10 \mu \mathrm{M}$ or $100 \mu \mathrm{M}$ roscovitine (Figure $4 \mathrm{~B}(\mathrm{~b}))$ and that roscovitine $(1 \mathrm{mg} / \mathrm{kg}-10 \mathrm{mg} / \mathrm{kg}$ - $50 \mathrm{mg} / \mathrm{kg}$ ) was not able to protect any of the mice against the electrically $6-\mathrm{Hz}$-induced seizures (40 mA stimulation current) (Figure 4C).

\section{Anticonvulsant actions of selective GSK-3 inhibition in zebrafish}

The ineffectiveness of roscovitine in these rodent models rather supports the hypothesis that indirubin exerts its anticonvulsant properties via GSK-3 inhibition. Selective GSK-3 inhibition has however never been directly tested in zebrafish or rodent seizure models. Therefore, to further strengthen our hypothesis, BIO-acetoxime (Figure 5A), a highly selective GSK-3 inhibitor was investigated. First, in the zebrafish PTZ seizure assay, analysis of the 1-hour tracking period revealed a significant decrease in the PTZ-induced movements when co-administered with 100 $\mu \mathrm{M}$ and $200 \mu \mathrm{M}$ (but not $30 \mu \mathrm{M}$ ) BIO-acetoxime (Figure 5B). This effect was seen from 5 minutes onwards. BIO-acetoxime $(30 \mu \mathrm{M}-100 \mu \mathrm{M}$ and $200 \mu \mathrm{M})$ alone did not affect the PTZ-induced seizures (Figure 5C). $\mathrm{LiCl}(0.05-0.5-5 \mathrm{mM})$ was not able to protect zebrafish larvae from PTZinduced seizures (see supplementary information file, Figure 1). 


\section{Target validation in zebrafish}

Next, we performed target validation experiments where we demonstrated that GSK-3 $\beta$ knockdown was able to counter PTZ-induced seizure behavior in a concentration dependent manner $(\mathrm{n}=52)$ (Figure 5D). We achieved targeted inhibition of GSK-3 $\beta$ by using antisensemediated morpholino (MO) knockdown in zebrafish embryos ${ }^{19-21}$. This shows that the lack of GSK-3 activity does show protective effects against PTZ-induced convulsions and that the antiseizure activity of BIO-acetoxime and indirubine might, at least to a certain extent, be mediated by GSK-3 inhibition.

\section{Anticonvulsant actions of selective GSK-3 inhibition in rodents}

To further validate the role of GSK-3 as a novel anticonvulsant target, we next investigated whether i.p. injection of BIO-acetoxime is able to change the seizure severity in a rat limbic seizure model. We showed that the pilocarpine-induced seizures are indeed significantly attenuated after systemic treatment of $0.5 \mathrm{mg} / \mathrm{kg}$ BIO-acetoxime (Figure 5E). Subsequently, in the electricallyinduced 6-Hz mouse model (48 mA stimulation current) BIO-acetoxime showed significant differences in seizure protection when sham-injected NMRI mice were compared with the animals receiving $0.05 \mathrm{mg} / \mathrm{kg}$ or $0.5 \mathrm{mg} / \mathrm{kg}$ (but not $5 \mathrm{mg} / \mathrm{kg}$ ) BIO-acetoxime (Figure $5 \mathrm{~F}(\mathrm{a})$ ). We also confirmed the anticonvulsant effects of BIO-acetoxime in two additional mouse strains, ie. FVB/N and $\mathrm{C} 57 \mathrm{Bl} / 6$ (Figure 5F(b) and 5F(c)) . 


\section{Selective GSK-3 inhibition is able to exert dose-dependent anticonvulsant effects in fully}

\section{kindled mice}

We also investigated whether GSK-3 inhibition could exert anticonvulsant actions in a clinically more relevant kindling model in which the brain has undergone long-term alterations that promote the epileptic state, without affecting blood-brain barrier permeability (based on i.v. injections of Fluorescein conjugated dextran $(3 \mathrm{kDa}, 30 \mathrm{mg} / \mathrm{Kg})$ - preliminary data not shown). Initially, $0.5 \mathrm{mg} / \mathrm{kg}$ BIO-acetoxime was tested in a batch of fully kindled "epileptic" mice (28 mA subconvulsive stimulation current, used throughout kindling). No differences were observed when the mean pretreatment seizure score was compared with the average post-treatment score (Figure 5G(a)). Next, systemic administration of $2.5 \mathrm{mg} / \mathrm{kg}$ or $5 \mathrm{mg} / \mathrm{kg}$ BIO-acetoxime was tested in two separate batches of 6-Hz fully kindled "epileptic" mice. Our results revealed a significant decrease in mean seizure score when the mean pre-treatment score of both experimental groups was compared with the post-treatment score (Figure $5 \mathrm{G}(\mathrm{b})$ and $5 \mathrm{G}(\mathrm{c})$ ).

\section{Discussion}

GSK-3, not CDK-5, is the likely anticonvulsant target of indirubin, the bioactive component of I. arrecta

In the present study we showed that indirubin, which was identified as the bioactive component of I. arrecta via bioassay-guided fractionation, exerts clear anticonvulsant effects in both zebrafish and rodent seizure models. Indirubin was shown to act on several cellular targets, of which the AhR and several protein kinases, including CDKs and GSK-3 ${ }^{22-25}$ seem to be the most prominent. Moreover, indirubin represents a novel structure when compared to the current ASDs and so far, 
no ASDs that target protein kinases have been identified. These criteria could be essential for its further development as a new potential ASD. Indirubin was initially discovered as the active ingredient of a plant mixture used in traditional Chinese medicine to treat chronic myelocytic leukaemia. As previously evidenced, the anti-proliferative effect of indirubin seems to be the result of the inhibition of CDKs ${ }^{22}$. In this sense, subsequent studies have identified indirubin as a potent inhibitor of CDK $-5^{23}$, although the compound is not devoid of activity towards other kinases ${ }^{22,23}$. Consequently, indirubin was also identified as a potent inhibitor of GSK-3 ${ }^{23}$. Pharmacological inhibitors of GSK-3 and some CDKs have a promising potential for applications against several neurodegenerative diseases such as Alzheimer's disease and Parkinson's disease ${ }^{23,}{ }^{26-28}$. Nevertheless, more clarification is needed concerning the role of these two neurobiological targets within the field of epilepsy.

Our results in zebrafish (cfr. Table 1) have pointed towards GSK-3 inhibition as the anticonvulsant mechanism of action of indirubin. Moreover, the anticonvulsant spectrum of the GSK-3 inhibitor BIO-acetoxime as presently demonstrated in the zebrafish, mouse and rat seizure models is identical to the results obtained for indirubin. Importantly, the affinity of BIO-acetoxime for CDK5 and CDK-2 (i.e. two CDKs that BIO-acetoxime can inhibit), is respectively 240 and 430 fold lower than its affinity for GSK-3 ${ }^{29}$. On the other hand, we demonstrated that the CDK-5 inhibitor roscovitine, although moderately effective in the PTZ zebrafish seizure assay, did not exhibit any anticonvulsant effects in the acute $6-\mathrm{Hz}$ mouse model and in the focal pilocarpine rat model - two seizure models in which indirubin and $\mathrm{BIO}$-acetoxime exerted both potent anticonvulsant effects. Taken together, our present observations thus suggest that the anticonvulsant actions of indirubin in the zebrafish, mouse and rat seizure models are most likely mediated via GSK-3 inhibtion, and not via CDK-5 inhibition. 


\section{Selective GSK-3 inhibition exerts potent anticonvulsant effects in acute seizure models and a chronic kindling model for epilepsy}

We next elaborated on the anticonvulsant effects of selective GSK-3 inhibition. For this purpose we compared the effects of indirubin with the effects obtained by BIO-acetoxime, a compound that is structurally related to indirubin and that, like indirubin, is a non-isoform specific GSK-3 inhibitor which is however $60 \mathrm{x}$ more potent. We here provided the first evidence that BIOacetoxime exerts clear anticonvulsant effects in acute- and chronic electrically-induced rodent models. Indeed, systemic administration of BIO-acetoxime was found to have potent anticonvulsant effects against acute 6-Hz-induced seizures. One should notice that in the 6-Hz model, we obtained a U-shaped dose-dependency curve. This is a phenomenon which is regularly seen when working with small molecules and might result from the fact that when higher concentrations of these molecules are used, other targets can be activated/inhibited or other offtarget effects might be evoked. Most interestingly, BIO-acetoxime also attenuated electricallyinduced seizures in kindled mice in a dose-dependent manner, indicating that GSK-3 inhibition can attenuate seizures even after brain excitability has been altered from normal, healthy conditions into an "epileptic" state. In addition, we demonstrated that BIO-acetoxime protects rats against focally-evoked pilocarpine-induced seizures. The given that BIO-acetoxime exerts antiseizure effects that are more potent than indirubine is not very surprising given the fact that the inhibitory activity of BIO-acetoxime towards GSK-3 is 60-fold higher than the inhibitory activity of indirubin (Table 1). GSK-3 inhibition however did not lower the seizure threshold in a mouse model in which PTZ was administered i.v. into the tail vein. Although the GSK-3 inhibitors were ineffective in the latter chemoconvulsant screening model, future investigation in additional, 
chronic models of epilepsy is needed. The positive results in the acute 6-Hz model, which is generally recognized as a model for refractory seizures, and the fact that BIO-acetoxime attenuates the seizures in fully-kindled mice, justify further research of GSK-3 as a potential new target for the treatment of pharmacoresistant seizures.

In this context, levetiracetam was novel in AED discovery in that sense that it was not discovered by the traditional screening approach. Indeed, it was found to be inactive against PTZ-induced seizures even at high, pharmacologically irrelevant doses ${ }^{30-32}$. However, in the kindled rat model, levetiracetam was found to be highly effective in blocking the fully expressed kindled seizures ${ }^{31-}$ 33. Later on, it became clear that levetiracetam exerts its anticonvulsant effects via a new, innovative mechanism of action, namely via binding to synaptic SV2A and thereby influencing synaptic vesicle exocytosis and neurotransmitter release ${ }^{34,35}$. In a way, the lack of efficacy of levetiracetam in traditional screening tests demonstrates the importance and utility of additional animal models, such as the electrically-induced models. Moreover, in light of the identification of levetiracetam, it can be concluded that the traditional acute screening tests are clearly not able to identify all targets, involved in seizure genesis and seizure suppression. Therefore, more chronic epilepsy models need to be implicated in the search for new ASDs. Indeed, chronic epilepsy models that are characterized by spontaneous, recurrent seizures and models for epileptogenesis will show molecular changes that are more connected to epilepsy than changes, evoked by acutelyinduced seizures in naïve animals.

\section{Is GSK-3 activation linked to enhanced seizure susceptibility?}

Since our results indicate that GSK-3 inhibition exerts anticonvulsant effects, it might be possible that epilepsy and epileptic disorders occur as a result of elevated GSK-3 levels. In this regard, 
increased GSK-3 activity has been linked to epilepsy and phenomena characterizing this disorder. For example, it has been shown that the absence of dopamine D2 receptors in the hippocampus leads to the activation of GSK-3 after systemic administration of kainic acid, resulting into a higher susceptibility to kainic acid-induced seizures and excitotoxicity of D2 null mice ${ }^{36,37}$. Furthermore, expression levels of GSK-3 were shown to increase after pilocarpine-induced status epilepticus in rats. Moreover, the same study showed that lovastatin, which is a cholesterol-lowering drug, reduces mossy fiber sprouting through reversal of these elevated GSK-3 levels ${ }^{38}$. In another study, Lohi and coworkers found that the phosphatase laforin, mutations in which induce Lafora Progressive Myoclonus epilepsy, activates GSK-3 ${ }^{39}$. Another interesting given is that blockage of the N-Methyl-D-aspartate receptors (NMDARs) inhibits the activity of GSK-3 ${ }^{40,41}$. In this regard it was shown that NMDA-mediated axonal impairment is alleviated after blocking the NMDARs and the consequent inhibition of GSK-3 $\beta$ activity ${ }^{40}$. Furthermore, it has been demonstrated that Particularly Interesting New Cysteine Histidine-rich (PINCH) protein, a highly conserved LIMdomain protein, and hyperphosphorylated Tau are significantly increased in epileptic tissue of patients with drug-resistant mesial temporal lobe epilepsy and that these changes are accompanied by decreased phosphorylation levels of GSK-3 ${ }^{42}$. Next, a study performed by Lui and coworkers showed, by means of Western Blot experiments, that refractory epilepsy patients have decreased

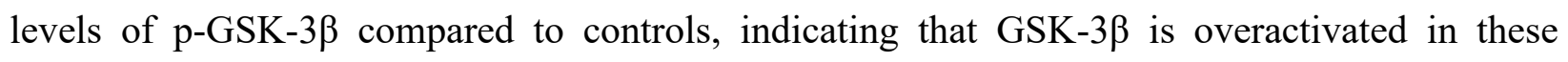
patients $^{40}$. Finally, one recent study investigated the effect of 4-benzyl-2-methyl-1,2,4thiadiazolidine-3,5-dione (TDZD-8), a non-ATP-competitive inhibitor of GSK-3 $\beta$ on kainic acidinduced seizures and neurodegeneration in mice. The results revealed that pretreatment with TDZD-8 was not able to protect the animals against the kainic acid-induced seizures. TDZD-8 did however significantly decrease the extent of neuronal damage in the pretreated mice ${ }^{43}$. 
Furthermore, the combined effect of a sub-effective dose of sodium valproate, a clinically used ASD with known GSK-3 inhibitory activity, with TDZD-8 was higher than that of TDZD-8 treatment alone ${ }^{43}$. On the other hand, a study by Lewerenz and coworkers showed that GSK-3 can be inhibited via phosphoinositide 3-kinase activation as a result of the activation of the Akt signaling pathway, which is induced by robust neuronal activity and in epileptic hippocampi ${ }^{44}$. Although the majority of these previously performed studies thus mainly suggest that increased seizure susceptibility might, at least to a certain extent, be the result of increased GSK-3 activity, our current study is the first to demonstrate the direct anticonvulsant effects of GSK-3 inhibition.

\section{GSK-3 inhibitors as potential new drugs for epilepsy treatment}

The close involvement of GSK-3 activity in diverse human pathologies has encouraged many researchers to develop inhibitors as therapeutic agents. To date, no selective GSK-3 inhibitors have been developed to treat epilepsy. Nevertheless, valproic acid (VPA), a sodium channel blocker which is used to treat bipolar disorder and epilepsy was shown to concentration-dependently inhibit GSK-3 ${ }^{45}$. We and others have previously reported antiseizure activity of VPA in zebrafish $\mathrm{PTZ}_{\text {model }}{ }^{1,6}$, and while VPA as also active in the mouse $6-\mathrm{Hz}$ model ${ }^{46}$, it is not in the rat pilocarpine $\operatorname{model}^{47}$. Also lamotrigine, another currently used ASD and sodium channel blocker can provide protection from GSK-3 facilitated apoptosis in human neuroblastoma cells ${ }^{48}$. The latter findings support the idea that modulation of GSK-3 activity may play a therapeutic beneficial role in epilepsy and epileptic disorders. The downstream mechanism of action that could be responsible for the anticonvulsive effects of GSK-3 inhibition in our hands remains unclear. Although we have shown with our target validation MO approach that GSK-3 $\beta$ knockdown leads to anticonvulsant effects, it would be very interesting to test whether indirubin and BIO-acetoxime 
also potentially interact with voltage-gated sodium channels (e.g. to exclude dual acting GSK-3 inhibition $/ \mathrm{Na}^{+}$channel inactivation as mechanism of action).

Future research on GSK-3 inhibitors in epilepsy, including the exploration of the effects of chronic GSK-3 inhibition is hence intriguing and mandatory.

\section{Methods}

\section{Bioassay-guided isolation of indirubin}

The leaves of I. arrecta were collected on Idjwi Island, Lake Kivu (Congo) and dried. For the testing in zebrafish, dried plant material was extracted with methanol. After centrifugation, clear supernatants were collected and concentrated using a rotavapor (Buchi Labortechnik, HendrikIdo-Ambacht, The Netherlands). The extract was suspended in DMSO before use (final concentration of $20 \mathrm{mg} / \mathrm{ml}$ ). For isolation of the active constituent the dried plant material was extracted multiple times with acetone. Extracts were combined, evaporated to dryness under reduced pressure on kieselguhr as a sorbent, and the resulting powder applied to the top of a silica gel 60 column. The fractionation was performed by liquid chromatography with ethylacetate as the mobile phase. Fractions containing the bioactive constituent were pooled and dried. The residue was dissolved in dichloromethane by heating and stored at $4{ }^{\circ} \mathrm{C}$ overnight. The precipitate contained the active constituent. Purity was determined by HPLC (diol column, mobile phase: ethylacetate) and was 85\% (as monitored at UV289). 


\section{Drugs and reagents}

Indirubin was obtained from Sigma-Aldrich Chemicals (Bornem, Belgium), BIO-acetoxime was purchased from Tocris Bioscience (Bristol, United Kingdom) and chosen because of its high inhibitory activity towards GSK-3, roscovitine from Selleckchem (Houston, USA), pilocarpine.HCl and PTZ from Sigma (St Louis, MO, USA). All other chemicals were analytical reagent grade and were obtained from Merck (Darmstadt, Germany). Aqueous solutions were prepared in fresh water purified by an arium pro UV system (Sartorius Stedim Biotech GmbH, Goettingen, Germany). Prior to their systemic administration in rodents, indirubin and BIOacetoxime were dissolved in propyleenglycol:saline (50:50). Since the metabolic and chemical stability of the compounds is unknown, solutions were freshly prepared and syringes (protected from light) were filled ex tempore to minimize the influence of possible degradation.

\section{Zebrafish}

Zebrafish (Danio rerio) with an $\mathrm{AB}$ or EK genetic background were maintained at $28.5^{\circ} \mathrm{C}$, on a 14/10 h light/dark cycle under standard aquaculture condition, and fertilized eggs were collected via natural spawning. Embryos were reared under constant light conditions in $0.3 \mathrm{x}$ Danieau's buffer, further referred to as 'embryo medium' - 1.5mM HEPES, $\mathrm{pH} 7.6,17.4 \mathrm{mM} \mathrm{NaCl}, 0.21 \mathrm{mM}$ $\mathrm{KCl}, 0.12 \mathrm{mM} \mathrm{MgSO}_{4}$, and $\left.0.18 \mathrm{mM} \mathrm{Ca}\left(\mathrm{NO}_{3}\right)_{2}\right)$ in an incubator at $28.5^{\circ} \mathrm{C}$. Although 10 days postfertilization (dpf) larvae would also be well-suited to identify blood-brain barrier permeable compounds, we here used larvae at 7 days post-fertilization dpf for the behavioral and electrographic assays. So far all CNS-active natural products that we have isolated from medicinal plants in our zebrafish screens, when tested in mice, have indeed shown activity in 7 dpf larvae ${ }^{12,}$ 
13. Targeted inhibition of GSK-3 $\beta$ was achieved using antisense-mediated MO knockdown in zebrafish embryos $^{19-21}$. For these MO injection embryos of the AB line were used.

\section{Toxicological evaluation}

Zebrafish larvae were incubated with compound or vehicle in the dark. After 90 minutes, each larva was individually checked under the microscope for the following signs of acute locomotor impairment: hypoactivity, decreased or no touch/escape response upon a light touch of the tail with a fine needle ${ }^{49,50}$, loss of posture, body deformation, exophthalmos (bulging of the eyes out of their sockets), slow or absent heartbeat, and death. After an overnight incubation (18 hours), assessment of larvae for the same above-mentioned signs of toxicity was repeated. A larva was considered normal if it could cover a distance twice its body length. A shorter distance travelled or movement in the same place was scored as a decreased or impaired touch response. No visible movement upon a touch stimulus was counted as no response. The maximum tolerated concentration (MTC) was thus defined as the maximum concentration that did not cause death and where not more than two out of 12 larvae exhibited any sign of locomotor impairment (including no touch response) after an 18 -hour incubation period.

\section{Behavioral analysis of anticonvulsant activity in zebrafish}

Six-dpf larvae were placed in a 96-well plate with one larva per well. The larvae were preincubated with compound or vehicle for 18 hours in a volume of $100 \mu$. The concentrations of compound used are described in the results section; for each experimental condition the final concentration of DMSO was $1 \% \mathrm{w} / \mathrm{v}$. After pre-incubation with each compound, embryo medium 
was added to twelve of the twenty-four larvae per treatment group. To the other twelve larvae, $100 \mu \mathrm{l}$ of PTZ solution was added to obtain a final concentration of $20 \mathrm{mM}^{12}$. Larvae were allowed to habituate for 5 minutes in a darkened chamber of an automated tracking device (ZebraBox, Viewpoint, France). The total locomotor activity was then measured using an automated tracking system (VideoTrack, Viewpoint, France) ${ }^{12}$. Total movement or activity was expressed in "actinteg" units. The actinteg value of Viewpoint software is defined as the sum of all image pixel changes detected during the time window defined for the experiment.

\section{Electroencephalographic analysis of anticonvulsant activity in zebrafish}

Each larva (6 dpf) was pre-incubated with either compound or vehicle for 18 hours in a volume of $400 \mu \mathrm{l}$ in individual wells of a 24-well plate. After the pre-incubation time, an equal volume of PTZ solution was added to the well to obtain a final concentration of $20 \mathrm{mM}$. After exposure for 15 minutes the larva was embedded in 2\% low-melting-point agarose. A glass electrode filled with artificial cerebrospinal fluid composed of (mM): $124 \mathrm{NaCl}, 2 \mathrm{KCl}, 2 \mathrm{MgSO}_{4}, 2 \mathrm{CaCl}_{2}, 1.25$

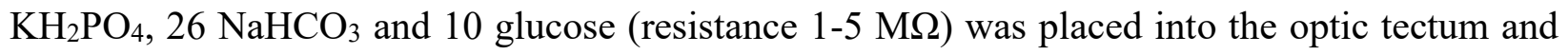
recording was performed in current clamp mode, low-pass filtered at 1-kHz, high-pass filtered 0.1Hz, digital gain 10, sampling interval 10 $\mu$ s (MultiClamp 700B amplifier, Digidata 1440A digitizer, both Axon instruments, USA). The EEG recordings started each time exactly 20 minutes after proconvulsant exposure and were continued for 10 minutes. Recordings from three to seven larvae were taken per experimental condition. Epileptiform activity was analyzed according to the duration of spiking paroxysms as described previously ${ }^{12}$. Briefly, we compared the total number, the average duration and the cumulative duration of epileptiform discharges in treated larvae. Spikes were categorized as interictal-like $(<3 \mathrm{~s})$ or ictal-like $(>3 \mathrm{~s}){ }^{51}$ with amplitudes exceeding 
three times the background noise with the aid of Clampfit 10.2 software (Molecular devices corporation, USA).

\section{Rodents}

All experiments were carried out according to the national guidelines on animal experimentation and were approved by the Ethical Committee for Animal Experiments of the Faculty of Medicine and Pharmacy of the Vrije Universiteit Brussel (Brussels, Belgium). The animals were kept under standard laboratory conditions, received food and water ad libitum and were kept on a 10/14 h dark/light cycle.

\section{Electrically-induced 6-Hz models in mice}

Male mice with NMRI, FVB/N and/or C57Bl/6 background (25-30g, Charles River, Chatillonsur-Chalaronne, France) were used to investigate the effects of the test compounds in the electrical 6-Hz refractory model. Prior to the electrical stimulation, $0.5 \%$ xylocaine was always applied to the cornea of the mice to induce local anesthesia and ensure good conductivity. Corneal stimulation ( $0.2 \mathrm{~ms}$ duration pulses at $6-\mathrm{Hz}$ for $3 \mathrm{~s})$ was administered by a constant current device (ECT Unit 57800; Ugo Basile, Comerio, Italy). Animals are restrained manually and released immediately in a cage of plexi glas following stimulation ${ }^{52,53}$.

The acutely evoked 6-Hz seizures were characterized by stun, forelimb clonus, twitching of vibrissae and/or Straub-tail for at least $45 \mathrm{~s}$. Each animal was tested once at a selected threshold current intensity which is defined as the current required to elicit clear seizures for at least $45 \mathrm{~s}$ in 6 consecutive naive animals. Protection is defined as the complete absence of seizures or when a 
seizure lasts for maximum $45 \mathrm{~s}$. This means that whenever seizures lasted $45 \mathrm{~s}$ or longer, animals were categorized in the non-protected group, implying that these animals could also show seizures that would last much longer than $45 \mathrm{~s}$. Male FVB/N mice were used to test the anticonvulsant effects of BIO-acetoxime in fully kindled mice. Unlike the acute 6-Hz model, the behavioral seizure severity in the $6-\mathrm{Hz}$ kindling model was scored based on Racine's scale ${ }^{54}$ : (1) facial twitches, (2) head nodding, (3) bilateral forelimb clonus, (4) rearing and (5) generalized clonus with rearing and falling. Mice were considered fully kindled when they have exhibited at least 10 consecutive generalized seizures (stages 3-5). The time required to reach the kindled state could differ from animal to animal. In general, we can state that all animals would be considered kindled after 10-15 days of daily stimulations (2x/day). To achieve the fully kindled state, mice were stimulated at a fixed subconvulsive threshold current twice daily, except the weekends, with a $4 \mathrm{~h}$ minimum interval between stimulations. Kindled mice were then stimulated for only 2 days a week, twice daily to maintain the fully kindled state. Meanwhile non-kindled mice were kept at the original stimulation scheme for maximum 1-2 more weeks. The anticonvulsant effect of BIOacetoxime was investigated in the "epileptic" fully kindled mice by stimulating these animals on Monday morning. On Monday afternoon, the animals were intraperitoneally (i.p.) injected with vehicle, 30 minutes prior to stimulation. On Tuesday morning, mice were again stimulated. On Tuesday afternoon, mice were injected i.p. with BIO-acetoxime and were stimulated 30 minutes later. Seizure severity was assessed using Racine's scale. The pre-treatment scores, calculated as the mean of the scores obtained during maintenance of the kindled state, were compared with the post-treatment scores, obtained after the treatment on Tuesday. To confirm any observed effects, the experiment was repeated, respecting one week of washout. During this washout week, all mice were stimulated for two days (twice daily) to maintain the kindled state. Compound testing during 
a longer period has not been validated so far. This means that only one dose of BIO-acetoxime could be tested in one batch of kindled mice. The latter explains why the number of animals differs for each tested dose (Figure 5G).

\section{Timed intravenous (i.v.) PTZ infusion seizure test in mice}

The threshold for different phases of PTZ-induced seizure activity was determined by i.v. infusion of PTZ $(7.5 \mathrm{mg} / \mathrm{ml})$ in the lateral tail vein of male NMRI mice at a constant rate of $150 \mu 1 / \mathrm{min}$. During the experiment, the animal could freely move in a plexi glas cage. The following end points were used to determine the seizure threshold: (1) first myoclonic twitch, (2) forelimb clonus, (3) falling (loss of righting reflexes), (4) tonic hindlimb extension (THE) and (5) death. Time was measured from the start of the infusion until the onset of these different stages. Seizure thresholds were determined for each animal according to the following equation: PTZ threshold dose (mg/kg) $=($ infusion rate $(\mathrm{ml} / \mathrm{s}) \times \mathrm{PTZ}$ concentration $(\mathrm{mg} / \mathrm{ml}) \times$ infusion duration $(\mathrm{s}) \times 1000) /$ weight of mouse (g).

\section{Chemically-induced focal seizure model in rats}

To test the anticonvulsant effects of our test compounds in the pilocarpine rat model, male albino Wistar rats (250-320 g) were used (Charles River, Chatillon-sur-Chalaronne, France). Microdialysis in rat hippocampus and concomittant seizure severity assessments using Racine's scale ${ }^{54}$ were performed as previously described ${ }^{55-57}$. The total seizure severity score (TSSS) for each animal was calculated as the sum of the highest seizure severity score attained in each of the 
collection periods following the start of pilocarpine administration, and was used as a measure of seizure severity. The protocols are depicted in Figures 2D, 4B and 5E.

\section{Beam-walking assay in mice}

The beam-walking assay was used to discover possible adverse effects such as problems with motor coordination or sedation, induced by the administration of indirubin. C57Bl/6 mice were trained to walk from a start platform along a ruler towards a closed box. The trained mice were administered compound or vehicle and were again tested on the beam. Mice that fell were returned to the position they fell from, with a maximum time of $60 \mathrm{~s}$ allowed on the beam. Measurements that were taken are time on beam, the number of foot slips (one or both hind limbs) and the number of falls ${ }^{58}$.

\section{Statistical analysis}

Statistical analyses were performed using GraphPad Prism 4.0 software package for Windows. Most of the data were normally distributed and consequently parametric statistical tests were used. All date are expressed as the mean value \pm SEM. For all analysis of the results obtained from the zebrafish experiments an unpaired student's t-test was used. One-way ANOVA, followed by the Bonferroni's post hoc test was used to compare the mean TSSSs obtained in the focal pilocarpine rat model and to compare the different seizure thresholds in the mouse PTZ i.v. model. For clarity of presentation, the results obtained in the acute $6-\mathrm{Hz}$ model are expressed as the $\%$ of protected versus $\%$ of non-protected mice and analysed with the chi-square test for trend. This test was followed by the Fisher's exact test to denote any significant differences between the experimental 
groups. To compare pre- and post-treatment seizure severity scores in the $6-\mathrm{Hz}$ corneally kindled mice, a non-parametric Mann-Whitney test was used. For all statistical analysis $\alpha$ was set at 0.05 .

\section{Author Contributions}

N.A. and I.S. designed the rodent experiments and the latter were performed by N.A. with support from L.W. All zebrafish experiments were designed by C.V.E, P.A.M.dW and A.D.C and performed by AS.K.S with support from T.A. N.A. wrote the manuscript with support from AS.K.S, I.S, A.D.C. and F.v.L. All authors provided critical feedback and helped to analyse the results and to shape the manuscript. JNC and PBB carried out ethnobotanical studies in DR Congo to identify I. arrecta as an anti-seizure medicinal plant, and identified, collected and processed I. arrecta plant material there; ARK identified, collected and processed I. arrecta plant material in Tanzania; RAEE and AIG carried out MS and NMR analyses to structurally identify indirubin.

\section{Conflict of Interest}

The authors declare that there is no conflict of interest regarding the publication of this manuscript.

\section{Funding Sources}

Vrije Universiteit Brussel (grant strategic research program SRP20) 


\section{Supporting information (Pdf)}

- No influence of $18 \mathrm{~h}$ pre-treatment of $\mathrm{LiCl}$ on PTZ-induced seizures in 6-dpf zebrafish 


\section{References}

1. Baraban, S. C., Taylor, M. R., Castro, P. A., and Baier, H. (2005) Pentylenetetrazole induced changes in zebrafish behavior, neural activity and c-fos expression, Neuroscience 131, 759-768.

2. Baraban, S. C., Dinday, M. T., Castro, P. A., Chege, S., Guyenet, S., and Taylor, M. R. (2007) A largescale mutagenesis screen to identify seizure-resistant zebrafish, Epilepsia 48, 1151-1157.

3. Baraban, S. C., and Löscher, W. (2014) What new modeling approaches will help us identify promising drug treatments?, Adv Exp Med Biol 813, 283-294.

4. Hortopan, G. A., Dinday, M. T., and Baraban, S. C. (2010) Zebrafish as a model for studying genetic aspects of epilepsy, Dis Model Mech 3, 144-148.

5. Vermoesen, K., Serruys, A. S., Loyens, E., Afrikanova, T., Massie, A., Schallier, A., Michotte, Y., Crawford, A. D., Esguerra, C. V., de Witte, P. A., Smolders, I., and Clinckers, R. (2011) Assessment of the convulsant liability of antidepressants using zebrafish and mouse seizure models, Epilepsy Behav 22, 450-460.

6. Afrikanova, T., Serruys, A. S., Buenafe, O. E., Clinckers, R., Smolders, I., de Witte, P. A., Crawford, A. D., and Esguerra, C. V. (2013) Validation of the zebrafish pentylenetetrazol seizure model: locomotor versus electrographic responses to antiepileptic drugs, PLoS One 8, e54166.

7. Suls, A., Jaehn, J. A., Kecskés, A., Weber, Y., Weckhuysen, S., Craiu, D. C., Siekierska, A., Djémié, T., Afrikanova, T., Gormley, P., von Spiczak, S., Kluger, G., Iliescu, C. M., Talvik, T., Talvik, I., Meral, C., Caglayan, H. S., Giraldez, B. G., Serratosa, J., Lemke, J. R., Hoffman-Zacharska, D., Szczepanik, E., Barisic, N., Komarek, V., Hjalgrim, H., Møller, R. S., Linnankivi, T., Dimova, P., Striano, P., Zara, F., Marini, C., Guerrini, R., Depienne, C., Baulac, S., Kuhlenbäumer, G., Crawford, A. D., Lehesjoki, A. E., de Witte, P. A., Palotie, A., Lerche, H., Esguerra, C. V., De Jonghe, P., Helbig, I., and Consortium, E. R. (2013) De novo loss-of-function mutations in CHD2 cause a fever-sensitive myoclonic epileptic encephalopathy sharing features with Dravet syndrome, Am J Hum Genet 93, 967-975.

8. Schubert, J., Siekierska, A., Langlois, M., May, P., Huneau, C., Becker, F., Muhle, H., Suls, A., Lemke, J. R., de Kovel, C. G., Thiele, H., Konrad, K., Kawalia, A., Toliat, M. R., Sander, T., Rüschendorf, F., Caliebe, A., Nagel, I., Kohl, B., Kecskés, A., Jacmin, M., Hardies, K., Weckhuysen, S., Riesch, E., Dorn, T., Brilstra, E. H., Baulac, S., Møller, R. S., Hjalgrim, H., Koeleman, B. P., Jurkat-Rott, K., Lehman-Horn, F., Roach, J. C., Glusman, G., Hood, L., Galas, D. J., Martin, B., de Witte, P. A., Biskup, S., De Jonghe, P., Helbig, I., Balling, R., Nürnberg, P., Crawford, A. D., Esguerra, C. V., Weber, Y. G., Lerche, H., and Consortium, E. R. (2014) Mutations in STX1B, encoding a presynaptic protein, cause fever-associated epilepsy syndromes, Nat Genet 46, 1327-1332.

9. Cragg, G. M., and Newman, D. J. (2013) Natural products: a continuing source of novel drug leads, Biochim Biophys Acta 1830, 3670-3695.

10. Moshi, M. J., Kagashe, G. A., and Mbwambo, Z. H. (2005) Plants used to treat epilepsy by Tanzanian traditional healers, J Ethnopharmacol 97, 327-336.

11. Sucher, N. J., and Carles, M. C. (2015) A pharmacological basis of herbal medicines for epilepsy, Epilepsy Behav 52, 308-318.

12. Orellana-Paucar, A. M., Serruys, A. S., Afrikanova, T., Maes, J., De Borggraeve, W., Alen, J., LeónTamariz, F., Wilches-Arizábala, I. M., Crawford, A. D., de Witte, P. A., and Esguerra, C. V. (2012) Anticonvulsant activity of bisabolene sesquiterpenoids of Curcuma longa in zebrafish and mouse seizure models, Epilepsy Behav 24, 14-22. 
13. Buenafe, O. E., Orellana-Paucar, A., Maes, J., Huang, H., Ying, X., De Borggraeve, W., Crawford, A. D., Luyten, W., Esguerra, C. V., and de Witte, P. (2013) Tanshinone IIA exhibits anticonvulsant activity in zebrafish and mouse seizure models, ACS Chem Neurosci 4, 1479-1487.

14. Challal, S., Buenafe, O. E., Queiroz, E. F., Maljevic, S., Marcourt, L., Bock, M., Kloeti, W., Dayrit, F. M., Harvey, A. L., Lerche, H., Esguerra, C. V., de Witte, P. A., Wolfender, J. L., and Crawford, A. D. (2014) Zebrafish bioassay-guided microfractionation identifies anticonvulsant steroid glycosides from the Philippine medicinal plant Solanum torvum, ACS Chem Neurosci 5, 993-1004.

15. Albertini, G., Walrave, L., Demuyser, T., Massie, A., De Bundel, D., and Smolders, I. (2018) 6 Hz corneal kindling in mice triggers neurobehavioral comorbidities accompanied by relevant changes in cFos immunoreactivity throughout the brain, Epilepsia 59, 67-78.

16. Liau, B. C., Jong, T. T., Lee, M. R., and Chen, S. S. (2007) LC-APCI-MS method for detection and analysis of tryptanthrin, indigo, and indirubin in daqingye and banlangen, J Pharm Biomed Anal 43, 346351.

17. Hu, J., Chang, H., Wang, L., Wu, S., Shao, B., Zhou, J., and Zhao, Y. (2008) Detection, occurrence and fate of indirubin in municipal sewage treatment plants, Environ Sci Technol 42, 8339-8344.

18. Cuong, N. M., Tai, B. H., and Hoan, D. H. (2010) Studies on the acetylation and NMR reassignment of indirubin derivatives, Nat Prod Res 24, 99-105.

19. Summerton, J., and Weller, D. (1997) Morpholino antisense oligomers: design, preparation, and properties, Antisense Nucleic Acid Drug Dev 7, 187-195.

20. Nasevicius, A., and Ekker, S. C. (2000) Effective targeted gene 'knockdown' in zebrafish, Nat Genet 26, 216-220.

21. Bill, B. R., Petzold, A. M., Clark, K. J., Schimmenti, L. A., and Ekker, S. C. (2009) A primer for morpholino use in zebrafish, Zebrafish 6, 69-77.

22. Hoessel, R., Leclerc, S., Endicott, J. A., Nobel, M. E., Lawrie, A., Tunnah, P., Leost, M., Damiens, E., Marie, D., Marko, D., Niederberger, E., Tang, W., Eisenbrand, G., and Meijer, L. (1999) Indirubin, the active constituent of a Chinese antileukaemia medicine, inhibits cyclin-dependent kinases, Nat Cell Biol $1,60-67$.

23. Leclerc, S., Garnier, M., Hoessel, R., Marko, D., Bibb, J. A., Snyder, G. L., Greengard, P., Biernat, J., Wu, Y. Z., Mandelkow, E. M., Eisenbrand, G., and Meijer, L. (2001) Indirubins inhibit glycogen synthase kinase-3 beta and CDK5/p25, two protein kinases involved in abnormal tau phosphorylation in Alzheimer's disease. A property common to most cyclin-dependent kinase inhibitors?, J Biol Chem 276, 251-260.

24. Kunikata, T., Tatefuji, T., Aga, H., Iwaki, K., Ikeda, M., and Kurimoto, M. (2000) Indirubin inhibits inflammatory reactions in delayed-type hypersensitivity, Eur J Pharmacol 410, 93-100.

25. Knockaert, M., Blondel, M., Bach, S., Leost, M., Elbi, C., Hager, G. L., Nagy, S. R., Han, D., Denison, M., Ffrench, M., Ryan, X. P., Magiatis, P., Polychronopoulos, P., Greengard, P., Skaltsounis, L., and Meijer, L. (2004) Independent actions on cyclin-dependent kinases and aryl hydrocarbon receptor mediate the antiproliferative effects of indirubins, Oncogene 23, 4400-4412.

26. Polychronopoulos, P., Magiatis, P., Skaltsounis, A. L., Myrianthopoulos, V., Mikros, E., Tarricone, A., Musacchio, A., Roe, S. M., Pearl, L., Leost, M., Greengard, P., and Meijer, L. (2004) Structural basis for the synthesis of indirubins as potent and selective inhibitors of glycogen synthase kinase- 3 and cyclindependent kinases, J Med Chem 47, 935-946.

27. Woodgett, J. R. (2001) Judging a protein by more than its name: GSK-3, Sci STKE 2001, re12. 
28. Bhat, R. V., Budd Haeberlein, S. L., and Avila, J. (2004) Glycogen synthase kinase 3: a drug target for CNS therapies, J Neurochem 89, 1313-1317.

29. Meijer, L., Skaltsounis, A. L., Magiatis, P., Polychronopoulos, P., Knockaert, M., Leost, M., Ryan, X. P., Vonica, C. A., Brivanlou, A., Dajani, R., Crovace, C., Tarricone, C., Musacchio, A., Roe, S. M., Pearl, L., and Greengard, P. (2003) GSK-3-selective inhibitors derived from Tyrian purple indirubins, Chem Biol 10, 1255-1266.

30. Löscher, W., and Hönack, D. (1993) Profile of ucb L059, a novel anticonvulsant drug, in models of partial and generalized epilepsy in mice and rats, Eur J Pharmacol 232, 147-158.

31. Löscher, W., Hönack, D., and Rundfeldt, C. (1998) Antiepileptogenic effects of the novel anticonvulsant levetiracetam (ucb L059) in the kindling model of temporal lobe epilepsy, $J$ Pharmacol Exp Ther 284, 474-479.

32. Klitgaard, H., Matagne, A., Gobert, J., and Wülfert, E. (1998) Evidence for a unique profile of levetiracetam in rodent models of seizures and epilepsy, Eur J Pharmacol 353, 191-206.

33. Matagne, A., and Klitgaard, H. (1998) Validation of corneally kindled mice: a sensitive screening model for partial epilepsy in man, Epilepsy Res 31, 59-71.

34. Kaminski, R. M., Matagne, A., Leclercq, K., Gillard, M., Michel, P., Kenda, B., Talaga, P., and Klitgaard, H. (2008) SV2A protein is a broad-spectrum anticonvulsant target: functional correlation between protein binding and seizure protection in models of both partial and generalized epilepsy, Neuropharmacology 54, 715-720.

35. Bialer, M., and White, H. S. (2010) Key factors in the discovery and development of new antiepileptic drugs, Nat Rev Drug Discov 9, 68-82.

36. Tripathi, P. P., Santorufo, G., Brilli, E., Borrelli, E., and Bozzi, Y. (2010) Kainic acid-induced seizures activate GSK-3 $\beta$ in the hippocampus of D2R-/- mice, Neuroreport 21, 846-850.

37. Dunleavy, M., Provenzano, G., Henshall, D. C., and Bozzi, Y. (2013) Kainic acid-induced seizures modulate Akt (SER473) phosphorylation in the hippocampus of dopamine D2 receptor knockout mice, $J$ Mol Neurosci 49, 202-210.

38. Lee, C. Y., Jaw, T., Tseng, H. C., Chen, I. C., and Liou, H. H. (2012) Lovastatin modulates glycogen synthase kinase- $3 \beta$ pathway and inhibits mossy fiber sprouting after pilocarpine-induced status epilepticus, PLoS One 7, e38789.

39. Lohi, H., Ianzano, L., Zhao, X. C., Chan, E. M., Turnbull, J., Scherer, S. W., Ackerley, C. A., and Minassian, B. A. (2005) Novel glycogen synthase kinase 3 and ubiquitination pathways in progressive myoclonus epilepsy, Hum Mol Genet 14, 2727-2736.

40. Liu, X., Ou, S., Yin, M., Xu, T., Wang, T., Liu, Y., Ding, X., Yu, X., Yuan, J., Huang, H., Zhang, X., Tan, X., Chen, L., and Chen, Y. (2017) N-methyl-D-aspartate receptors mediate epilepsy-induced axonal impairment and tau phosphorylation via activating glycogen synthase kinase- $3 \beta$ and cyclin-dependent kinase 5, Discov Med 23, 221-234.

41. De Sarno, P., Bijur, G. N., Zmijewska, A. A., Li, X., and Jope, R. S. (2006) In vivo regulation of GSK3 phosphorylation by cholinergic and NMDA receptors, Neurobiol Aging 27, 413-422.

42. Liu, C., Russin, J., Heck, C., Kawata, K., Adiga, R., Yen, W., Lambert, J., Stear, B., Law, M., Marquez, Y., Crino, P., Millett, D., and Langford, D. (2017) Dysregulation of PINCH signaling in mesial temporal epilepsy, J Clin Neurosci 36, 43-52.

43. Bhowmik, M., Khanam, R., Saini, N., and Vohora, D. (2015) Activation of AKT/GSK3 $\beta$ pathway by TDZD-8 attenuates kainic acid induced neurodegeneration but not seizures in mice, Neurotoxicology 46, 44-52. 
44. Lewerenz, J., Baxter, P., Kassubek, R., Albrecht, P., Liefferinge, J. V., Westhoff, M. A., Halatsch, M. E., Karpel-Massler, G., Meakin, P. J., Hayes, J. D., Aronica, E., Smolders, I., Ludolph, A. C., Methner, A., Conrad, M., Massie, A., Hardingham, G. E., and Maher, P. (2014) Phosphoinositide 3-Kinases Upregulate System xc(-) via Eukaryotic Initiation Factor $2 \alpha$ and Activating Transcription Factor 4 - A Pathway Active in Glioblastomas and Epilepsy, Antioxid Redox Signal.

45. Chen, G., Huang, L. D., Jiang, Y. M., and Manji, H. K. (1999) The mood-stabilizing agent valproate inhibits the activity of glycogen synthase kinase-3, J Neurochem 72, 1327-1330.

46. Barton, M. E., Klein, B. D., Wolf, H. H., and White, H. S. (2001) Pharmacological characterization of the $6 \mathrm{~Hz}$ psychomotor seizure model of partial epilepsy, Epilepsy Res 47, 217-227.

47. Klitgaard, H., Matagne, A., Grimee, R., Vanneste-Goemaere, J., and Margineanu, D. G. (2003) Electrophysiological, neurochemical and regional effects of levetiracetam in the rat pilocarpine model of temporal lobe epilepsy, Seizure 12, 92-100.

48. Li, X., Bijur, G. N., and Jope, R. S. (2002) Glycogen synthase kinase-3beta, mood stabilizers, and neuroprotection, Bipolar Disord 4, 137-144.

49. Fetcho, J. R., Higashijima, S., and McLean, D. L. (2008) Zebrafish and motor control over the last decade, Brain Res Rev 57, 86-93.

50. Pietri, T., Manalo, E., Ryan, J., Saint-Amant, L., and Washbourne, P. (2009) Glutamate drives the touch response through a rostral loop in the spinal cord of zebrafish embryos, Dev Neurobiol 69, 780-795. 51. D'Antuono, M., Köhling, R., Ricalzone, S., Gotman, J., Biagini, G., and Avoli, M. (2010) Antiepileptic drugs abolish ictal but not interictal epileptiform discharges in vitro, Epilepsia 51, 423-431. 52. De Bundel, D., Schallier, A., Loyens, E., Fernando, R., Miyashita, H., Van Liefferinge, J., Vermoesen, K., Bannai, S., Sato, H., Michotte, Y., Smolders, I., and Massie, A. (2011) Loss of System xFormula Does Not Induce Oxidative Stress But Decreases Extracellular Glutamate in Hippocampus and Influences Spatial Working Memory and Limbic Seizure Susceptibility., J Neurosci 31, 5792-5803.

53. Kaminski, R. M., Livingood, M. R., and Rogawski, M. A. (2004) Allopregnanolone analogs that positively modulate GABA receptors protect against partial seizures induced by 6-Hz electrical stimulation in mice, Epilepsia 45, 864-867.

54. Racine, R. (1972) Modification of seizure activity by electrical stimulation. II. Motor seizure., Electroencephalogr Clin Neurophysiol 32, 281-294.

55. Aourz, N., De Bundel, D., Stragier, B., Clinckers, R., Portelli, J., Michotte, Y., and Smolders, I. (2011) Rat hippocampal somatostatin sst3 and sst4 receptors mediate anticonvulsive effects in vivo: indications of functional interactions with sst2 receptors., Neuropharmacology 61, 1327-1333.

56. Aourz, N., Portelli, J., Coppens, J., De Bundel, D., Di Giovanni, G., Van Eeckhaut, A., Michotte, Y., and Smolders, I. (2014) Cortistatin-14 mediates its anticonvulsant effects via sst 2 and sst3 but not ghrelin receptors, CNS Neurosci Ther 20, 662-670.

57. De Bundel, D., Fafouri, A., Csaba, Z., Loyens, E., Lebon, S., El Ghouzzi, V., Peineau, S., Vodjdani, G., Kiagiadaki, F., Aourz, N., Coppens, J., Walrave, L., Portelli, J., Vanderheyden, P., Chai, S. Y., Thermos, K., Bernard, V., Collingridge, G., Auvin, S., Gressens, P., Smolders, I., and Dournaud, P. (2015) Trans-Modulation of the Somatostatin Type 2A Receptor Trafficking by Insulin-Regulated Aminopeptidase Decreases Limbic Seizures, J Neurosci 35, 11960-11975.

58. Stanley, J. L., Lincoln, R. J., Brown, T. A., McDonald, L. M., Dawson, G. R., and Reynolds, D. S. (2005) The mouse beam walking assay offers improved sensitivity over the mouse rotarod in determining motor coordination deficits induced by benzodiazepines, J Psychopharmacol 19, 221-227. 


\section{$\underline{\text { Table Legends }}$}

Table 1: Maximal Tolerated Concentrations (MTC) values, IC50 values and results of the behavioral PTZ assay in zebrafish. The activity in the zebrafish (ZF) PTZ assay is depicted as -, ,+++ or +++ for non active, significantly active with $\mathrm{p}<0.05, \mathrm{p}<0.01$ and $\mathrm{p}<0.001$, respectively.

\section{Figure Legends}

Figure 1: Anticonvulsant activity of Congolese methanolic plant extracts and TLC separation of constituents of Indigofera arrecta. (A) Ability of seven medicinal plant extracts to suppress convulsive behavior in the larval zebrafish PTZ seizure assay. Extract 3 corresponds to Indigofera arrecta. (B) Anticonvulsant activity identified for band (fraction) number 3. $[* \mathrm{P}<0.05 ; * * \mathrm{P}<0.01 ; * * * \mathrm{P}<0.001]$. (C) Structural formula of Indirubin.

Figure 2: Anticonvulsant activities of indirubin in zebrafish, mouse and rat seizure models. (A) Behavioral response of indirubin $(30 \mu \mathrm{M}, 100 \mu \mathrm{M}$ and $300 \mu \mathrm{M})$ in zebrafish larvae. Values significantly different from corresponding PTZ control are marked. Total locomotor activity is expressed in actinteg units and averaged for 5-min intervals. The number of larvae tested for each concentration is $\mathrm{n}=24$. (B) $30 \mu \mathrm{M}, 100 \mu \mathrm{M}$ and $300 \mu \mathrm{M}$ of indirubin alone does not influence the locomotor activity in the zebrafish larvae. The number of larvae tested for each concentration is also for these groups $n=24$. (C) Indirubin suppresses PTZ-induced epileptiform discharges as demonstrated by (a) the number of interictal-like spikes, (b) number of ictal-like spikes and (c) total cumulative duration of epileptiform activity (ictal and inter-ictal activity) measured. The number of recordings analyzed were: Vehicle (n=10), PTZ $20 \mathrm{mM}(\mathrm{n}=14)$ and Indirubin $300 \mu \mathrm{M}$ + PTZ $20 \mathrm{mM}$ (n=8). (D) (a) Microdialysis protocol. (b) Indirubin prevented seizures in the focal pilocarpine rat model. [ $\left.{ }^{*} \mathrm{P}<0.05 ; * * \mathrm{P}<0.01 ; * * * \mathrm{P}<0.001\right]$. (E). Anticonvulsant actions of indirubin against 6-Hz-induced electrical seizures in mice (stimulation current $=53 \mathrm{~mA}$ ).

Figure 3: Indirubin does not alter motor coordination in the mouse beamwalking test. Performance of mice in the beamwalking test after treatment with (A) indirubin or (B) diazepam. The y-axis denotes (a) time on beam (in seconds), (b) number of footslips or (c) number of falls. The $\mathrm{x}$-axis denotes the treatment dose (1 mg/kg diazepam; $10 \mathrm{mg} / \mathrm{kg}$ indirubin). Vehicle used was phosphate buffered saline (PBS) and PEG400/H2O for diazepam and indirubin respectively. 
Number of animals used was $\mathrm{n}=3$ for Vehicle and diazepam and $\mathrm{n}=6$ for indirubin. $[* \mathrm{P}<0.05 ; * * \mathrm{P}<0.01]$

Figure 4: Roscovitine does not alter seizure severity in an acute rat and mouse model for epileptic seizures. (A) Structural formula of roscovitine. (B) (a) Microdialysis protocol. Roscovitine is inactive in (B) (b) the pilocarpine rat model and (C) the acute 6-Hz model for refractory seizures (40 mA stimulation current).

\section{Figure 5: Anticonvulsant activities of BIO-acetoxime in zebrafish, mouse and rat seizure} models. (A) Structural formula of BIO-acetoxime. (B) BIO-acetoxime was tested at three different concentrations: $30 \mu \mathrm{M}, 100 \mu \mathrm{M}$ and $200 \mu \mathrm{M}$. Total locomotor activity is expressed in actinteg units and averaged for 5-min intervals. Values significantly different from corresponding PTZ control are marked. The number of larvae tested for each concentration is $n=24$. (C) $30 \mu \mathrm{M}, 100$ $\mu \mathrm{M}$ and $200 \mu \mathrm{M}$ BIO-acetoxime alone did not influence the locomotor activity in the zebrafish larvae. The number of larvae tested for each concentracion is n=24 (D) Graph denotes total larval activity (expressed in actinteg units), averaged for 10-min intervals over a period of 60 minutes. All time intervals were compared to the corresponding PTZ control using the unpaired Student's t-test. Values that were significantly different from PTZ control are marked with $*(\mathrm{p}<0.05)$ or $* * *$ $(\mathrm{p}<0.001)$. Standard deviations are only shown for uninjected embryos + PTZ for visual clarity. The number of larvae tested for each concentration is: $\mathrm{n}=52$ for uninjected, uninjected + PTZ, 200 $\mu \mathrm{M}$ MO, $200 \mu \mathrm{M} \mathrm{MO}+\mathrm{PTZ}$ and $\mathrm{n}=48$ for $300 \mu \mathrm{M}$ MO, $300 \mu \mathrm{M} \mathrm{MO}+\mathrm{PTZ}$. (E) (a) Anticonvulsant effects of BIO-acetoxime $(0.5 \mathrm{mg} / \mathrm{kg})$ in the focal pilocarpine rat model. (b) Microdialysis protocol. (F) Anticonvulsant effects of BIO-acetoxime in the acute 6-Hz mouse model in (a) NMRI (48 mA stimulation current), (b) FVB/N (40 mA stimulation current) and (c) C57Bl/6 (52 mA stimulation current) mice. (G) Anticonvulsant effects of (a) $0.5 \mathrm{mg} / \mathrm{kg}$, (b) 2.5 $\mathrm{mg} / \mathrm{kg}$ and (c) $5 \mathrm{mg} / \mathrm{kg}$ BIO-acetoxime in 6-Hz fully kindled FVB/N mice (28 mA subconvulsive stimulation current). [*P $<0.05 ; * * \mathrm{P}<0.01 ; * * * \mathrm{P}<0.001]$. 